\section{Adaptación y validación de instrumentos para medir errores en las recetas médicas y en el proceso de preparación de medicamentos en farmacia de pacientes ambulatorios}

\author{
CAROLINA ALVARADO A. ${ }^{1, a}$, XIMENA OSSA G. ${ }^{2, \mathrm{~b}}$, LUIS BUSTOS M., ${ }^{2, \mathrm{c}}$
}

Background: Adverse effects of medications are an important source of morbidity. Prescription and dispensing errors are an important cause of these adverse effects. Aim: To adapt and validate two checklists, one to measure errors in handwritten prescriptions and other to detected errors in the medication dispensing process of hospital pharmacies for outpatient care. Material and Methods: The study was conducted in three stages. First, checklists for medication errors developed elsewhere were adapted. Afterwards, the checklists were reviewed by experts. Finally, the inter and intra-observer reliability of each checklist was assessed, testing them in 32 occasions by two independent observers. Results: The checklists for medication prescription and dispensing were composed by 12 and seven items, respectively. They were corrected according to experts' opinions. The intraclass correlations of the results of each tester were 0.68 and 0.82 for the prescription and dispensing error checklists, respectively. Conclusions: The developed checklists for the detection of errors in prescription and dispensing of medications are reliable en can be applied in future studies.

(Rev Med Chile 2014; 142: 1547-1552)

Key words: Medical errors; Reproducibility of results; Validation studies.

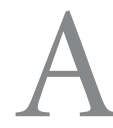

ctualmente es conocido y aceptado que el uso de medicamentos puede conducir a riesgos que pueden tener consecuencias negativas en los pacientes. Además de la presentación de reacciones adversas propias de cada medicamento utilizado en condiciones adecuadas, también se producen numerosos efectos nocivos por errores durante su proceso de utilización clínica, lo que denominamos como "errores de medicación”. Las investigaciones publicadas indican que aproximadamente 1,8\% de los pacientes hospitalizados sufre acontecimientos adversos originados por errores de medicación, y que cada

\author{
'Servicio de Farmacia. Hospital \\ Nueva Imperial, Servicio de Salud \\ Araucanía Sur, Nueva Imperial, \\ Chile. \\ ${ }^{2}$ Centro de Investigación y \\ Gestión en Salud (CIGES) \\ y Departamento de Salud \\ Pública y Facultad de Medicina, \\ Universidad de La Frontera, \\ Temuco, Chile. \\ aQuímico Farmacéutico.

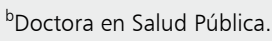 \\ 'Bioestadístico.
Conflictos de intereses: L. Bustos ha recibido honorarios de los laboratorios Astra Zeneca S.A. y Bristol-Meyers-Squibb en otros estudios no relacionados con el actual. C. Alvarado y X. Ossa declararon no tener conflictos de \\ intereses.
}

Recibido el 23 de junio de 2014, aceptado el 13 de noviembre de 2014.

Correspondencia a:

Carolina Alvarado.

caalvarado@gmail.com acontecimiento adverso alarga la estadía hospitalaria en aproximadamente 4,6 días ${ }^{1}$.

The National Coordinating Council for Medication Error Reporting and Prevention (NCCMERP) define un error de medicación como "cualquier incidente prevenible que puede causar daño al paciente o dar lugar a una utilización inapropiada de los medicamentos, cuando estos están bajo control de profesionales sanitarios o del paciente o consumidor" . Este error de medicación puede ocurrir en cualquier etapa del proceso de utilización de los medicamentos, por lo que los métodos de detección más adecuados no siempre son los 
Validación de instrumentos para medir errores de medicación - C. Alvarado et al

mismos. Algunos estudios consideran que el método más sensible para detectar errores en la dispensación y administración de medicamentos es la observación, y que la revisión de expedientes sería el más adecuado para detectar errores en las prescripciones médicas ${ }^{3}$. Si bien existen clasificaciones internacionales de errores de medicación, las definiciones de los errores y los métodos de detección, más que ser reproducibles y fiables, están más bien sujetos a las preferencias de cada investigador, lo que provoca grandes dificultades en la comparación de datos de diferentes estudios ${ }^{3,4}$.

Los errores en las recetas médicas son fallas en el proceso de escritura de éstas, que dan lugar a una instrucción errónea acerca de una o más de las "características normales" de una receta, entendiéndose por esto a la identidad del paciente, la identidad de la droga, la formulación, dosis, vía, tiempo, frecuencia y duración de la administración ${ }^{5}$. Por otra parte, un error de dispensación de medicamentos se define como la discrepancia entre lo que indica una receta médica y el medicamento dispensado por la farmacia al paciente o que distribuye a la sala de hospitalización sobre la base de esta receta ${ }^{6}$. Si este error es detectado antes que los medicamentos lleguen al paciente se le denomina "dispensing near miss", porque es un error que se pesquisa en el proceso de preparación de las recetas, antes de entregar los medicamentos al paciente y evita daño innecesario ${ }^{7}$.

En Chile, el uso de sistemas informáticos para la prescripción y dispensación de recetas en las diversas unidades de farmacia del país, tanto de la red pública como privada, presentan diferentes realidades. Se pueden encontrar establecimientos de salud que cuentan con programas de recetas electrónicas más desarrollados unos que otros, a veces sólo implementados en atención de pacientes hospitalizados o atención ambulatoria, o en ambos. Existen establecimientos de salud que no cuentan con este tipo de tecnología, y una proporción importante de sus recetas, o en su totalidad, son realizadas en forma manuscrita por los médicos.

Este escenario ha generado una alta necesidad de estudiar los errores de medicación y con ello, la búsqueda de métodos que permitan realizar estudios con instrumentos que puedan reproducir en forma fiable los resultados.

El objetivo de este estudio fue adaptar y validar dos pautas de chequeo, una que permita medir errores en las recetas médicas manuscritas y, otra, errores en el proceso de preparación de medicamentos en farmacias de atención de pacientes ambulatorios, de fácil entendimiento y aplicación. Este trabajo constituye la primera parte de un estudio para medir prevalencia de errores en las recetas médicas y en el proceso de preparación que se llevó a cabo en la farmacia de pacientes ambulatorios del Hospital de Nueva Imperial.

\section{Material y Método}

Estudio de corte transversal realizado en el Hospital de Nueva Imperial. Este establecimiento es de mediana complejidad con una capacidad de 135 camas. Cuenta con un Servicio de Farmacia que realiza dispensación de medicamentos a pacientes hospitalizados y ambulatorios (pacientes con alta médica, atendidos en policlínicos y Servicio de Urgencia) en forma independiente. El hospital no cuenta aún con un sistema de receta electrónica por lo que los facultativos deben realizar en forma manuscrita sus prescripciones. Este estudio fue realizado en la Sección de Farmacia de pacientes ambulatorios, donde ocurre la mayor dispensación de recetas.

Se realizó una adaptación y validación de dos pautas de chequeo en tres etapas: proceso de adaptación en primera instancia, validación a través de opiniones de expertos, en una segunda fase y, por último, mediciones de confiabilidad con pruebas estadísticas aplicadas a cada instrumento.

\section{Primera etapa}

Adaptación de pautas de chequeo: Dos pautas de chequeo fueron adaptadas para medir errores en las recetas médicas prescritas manualmente, $\mathrm{y}$ para el proceso de preparación en farmacia de los medicamentos indicados en las recetas. Ambas pautas se basaron en la clasificación de errores de medicación del grupo de trabajo Ruiz-Jarabo $2000^{8}$, lo que, a su vez, corresponde a una adaptación de la clasificación de errores del NCCMERP a la práctica española. Esta clasificación incluye categorías y subcategorías básicas necesarias para detectar, analizar y registrar errores de medicación. Este estudio se basó en el apartado número cinco ("Características del error") de dicha clasificación, específicamente en la categoría "Tipos de error”. Para la construcción de los diferentes 
ítems de cada pauta de chequeo se seleccionaron aquellos que consideraran los siguientes criterios:

- Ítems que tenían relación con errores específicamente en las recetas y procesos de preparación de la cadena terapéutica.

- Ítems referentes a tipificación de errores y no aquellos que se referían a causas o consecuencias de éstos.

- Aquellos referidos a detección observacional de los errores y que no implicara intervención.

Además, se consideraron los requisitos que deben cumplir las recetas médicas de acuerdo al reglamento chileno 9 .

\section{Segunda etapa}

Opinión de expertos: Se sometió ambos instrumentos a la opinión de cuatro profesionales Químicos Farmacéuticos con amplia experiencia en el área de farmacia asistencial, y que se desempeñaban, al momento de la consulta, en los hospitales: Victoria, Dr. Juan Hepp Dubiau (Purranque), Hospital Base Valdivia y Dr. Augusto Riffart (Castro). La información se recopiló mediante una pauta que recogía sus percepciones con respecto a la pertinencia de cada ítem, la comprensión en la redacción, observaciones y sugerencias.

\section{Tercera etapa}

Medición de confiabilidad: Una vez realizados los cambios sugeridos por los expertos, se efectuaron 16 mediciones de errores con cada pauta, lo que corresponde a $10 \%$ del promedio diario de recetas (aproximadamente 160 recetas) y de sus respectivas preparaciones, durante dos días. Estas mediciones fueron ejecutadas por dos observadores independientes. Los datos fueron introducidos en una base de Excel y luego trasladados al paquete estadístico Stata $11^{\circledR}$, donde se analizó para ambas, la confiabilidad interevaluador ítem por ítem por medio de la prueba estadísticas de McNemar y el test de correlación intraclase para evaluar la concordancia general de cada instrumento.

El estudio fue autorizado por el Comité de ética del Servicio de Salud Araucanía Sur y la anuencia de las autoridades del Hospital de Nueva Imperial.

\section{Resultados}

De la primera etapa (adaptación de pautas de chequeo): Las pautas de chequeo quedaron con- formadas por 12 ítems para medir errores en las recetas médicas y 7 ítems para medir errores en las preparaciones de recetas.

De la segunda etapa (opinión de expertos): Las modificaciones sugeridas dieron como resultado la mantención del número de ítems en cada pauta de chequeo, y mejoras en la redacción de cada ítem.

De la tercera etapa (medición de confiabilidad): Se realizaron 32 mediciones para cada pauta, ejecutadas por dos observadores independientes. En la pauta de 12 ítems, en 11 no se encontraron diferencias significativas entre ambas observaciones, $y$ al aplicar el test de correlación intraclase sobre la puntuación total, dio un valor de 0,57. Ante esto, se realizaron algunos cambios al instrumento en relación a la redacción de los ítems, y se llevaron a cabo nuevamente las 32 mediciones por dos observadores independientes, lo que resultó finalmente en que los ítems no presentaran diferencias significativas (Tabla 1), y el test de correlación intraclase aumentó su valor a 0,68.

Para la pauta de chequeo de 7 ítems, la evaluación no mostró diferencias significativas en las 32 mediciones de ambos observadores (Tabla 2), y el valor del test de correlación intraclase fue de 0,82 . Por lo tanto, no fue necesaria una nueva evaluación.

Construcción final de la pauta de chequeo: Cada pauta elaborada quedó conformada finalmente con igual número de ítems que al inicio tanto para medir errores en las recetas médicas como para medir errores en las preparaciones de recetas al término de este período.

A continuación se describen los ítems y lo que representa cada uno de ellos en ambos instrumentos:

\section{Tipo de errores en la receta médica}

A. No es posible determinar identificación del paciente, cuando existe ausencia de los datos del paciente, estén ilegibles o incompletos.

B. No es posible determinar concentración del medicamento, cuando existe ausencia de la concentración del o los medicamentos contenidos en ella, la información sea ilegible o esté incompleta.

C. No es posible determinar la forma farmacéutica del medicamento, cuando existe ausencia de la forma farmacéutica del o los medicamentos contenidos en ella, la información sea ilegible o esté incompleta. 
Validación de instrumentos para medir errores de medicación - C. Alvarado et al

Tabla 1. Medición de confiabilidad en pauta de chequeo de errores en receta médica

\begin{tabular}{|c|c|c|c|c|c|c|c|}
\hline Ítem & Evaluador 1 & Con Error & $\begin{array}{l}\text { 1ra medición } \\
\text { Evaluador } 2 \\
\text { Sin Error }\end{array}$ & $\mathbf{p}$ & Con Error & $\begin{array}{l}\text { 2da medición } \\
\text { Evaluador } 2 \\
\text { Sin Error }\end{array}$ & $\mathbf{p}$ \\
\hline A & $\begin{array}{l}\text { Con error } \\
\text { Sin error }\end{array}$ & $\begin{array}{l}2 \\
0\end{array}$ & $\begin{array}{r}0 \\
30\end{array}$ & 1,0000 & $\begin{array}{l}4 \\
1\end{array}$ & $\begin{array}{r}0 \\
27\end{array}$ & 1,0000 \\
\hline B & $\begin{array}{l}\text { Con error } \\
\text { Sin error }\end{array}$ & $\begin{array}{l}3 \\
0\end{array}$ & $\begin{array}{r}6 \\
23\end{array}$ & 0,0313 & $\begin{array}{l}2 \\
0\end{array}$ & $\begin{array}{r}0 \\
30\end{array}$ & 1,0000 \\
\hline C & $\begin{array}{l}\text { Con error } \\
\text { Sin error }\end{array}$ & $\begin{array}{r}28 \\
0\end{array}$ & $\begin{array}{l}0 \\
4\end{array}$ & 1,0000 & $\begin{array}{l}0 \\
0\end{array}$ & $\begin{array}{r}0 \\
32\end{array}$ & 1,0000 \\
\hline D & $\begin{array}{l}\text { Con error } \\
\text { Sin error }\end{array}$ & $\begin{array}{r}26 \\
0\end{array}$ & $\begin{array}{l}3 \\
3\end{array}$ & 0,2500 & $\begin{array}{l}0 \\
0\end{array}$ & $\begin{array}{r}0 \\
32\end{array}$ & 1,0000 \\
\hline $\mathrm{E}$ & $\begin{array}{l}\text { Con error } \\
\text { Sin error }\end{array}$ & $\begin{array}{l}0 \\
4\end{array}$ & $\begin{array}{r}1 \\
27\end{array}$ & 0,3750 & $\begin{array}{l}2 \\
0\end{array}$ & $\begin{array}{r}2 \\
28\end{array}$ & 0,5000 \\
\hline $\mathrm{F}$ & $\begin{array}{l}\text { Con error } \\
\text { Sin error }\end{array}$ & $\begin{array}{r}10 \\
0\end{array}$ & $\begin{array}{r}3 \\
19\end{array}$ & 0,2500 & $\begin{array}{l}8 \\
5\end{array}$ & $\begin{array}{r}4 \\
15\end{array}$ & 1,0000 \\
\hline G & $\begin{array}{l}\text { Con error } \\
\text { Sin error }\end{array}$ & $\begin{array}{l}0 \\
0\end{array}$ & $\begin{array}{r}3 \\
29\end{array}$ & 0,2500 & $\begin{array}{l}1 \\
0\end{array}$ & $\begin{array}{r}0 \\
31\end{array}$ & 1,0000 \\
\hline $\mathrm{H}$ & $\begin{array}{l}\text { Con error } \\
\text { Sin error }\end{array}$ & $\begin{array}{l}0 \\
0\end{array}$ & $\begin{array}{r}0 \\
32\end{array}$ & 1,0000 & $\begin{array}{l}0 \\
0\end{array}$ & $\begin{array}{r}0 \\
32\end{array}$ & 1,0000 \\
\hline I & $\begin{array}{l}\text { Con error } \\
\text { Sin error }\end{array}$ & $\begin{array}{l}5 \\
0\end{array}$ & $\begin{array}{r}0 \\
27\end{array}$ & 1,0000 & $\begin{array}{l}8 \\
0\end{array}$ & $\begin{array}{r}1 \\
23\end{array}$ & 1,0000 \\
\hline J & $\begin{array}{l}\text { Con error } \\
\text { Sin error }\end{array}$ & $\begin{array}{l}0 \\
0\end{array}$ & $\begin{array}{r}1 \\
31\end{array}$ & 1,0000 & $\begin{array}{l}0 \\
0\end{array}$ & $\begin{array}{r}2 \\
30\end{array}$ & 0,5000 \\
\hline K & $\begin{array}{l}\text { Con error } \\
\text { Sin error }\end{array}$ & $\begin{array}{r}16 \\
1\end{array}$ & $\begin{array}{r}0 \\
15\end{array}$ & 1,0000 & $\begin{array}{r}13 \\
0\end{array}$ & $\begin{array}{r}0 \\
19\end{array}$ & 1,0000 \\
\hline L & $\begin{array}{l}\text { Con error } \\
\text { Sin error }\end{array}$ & $\begin{array}{l}0 \\
0\end{array}$ & $\begin{array}{r}0 \\
32\end{array}$ & 1,0000 & $\begin{array}{l}0 \\
0\end{array}$ & $\begin{array}{r}0 \\
32\end{array}$ & 1,0000 \\
\hline
\end{tabular}

Prueba de McNemar.

Tabla 2. Medición de confiabilidad en pauta de chequeo de errores en preparación de recetas

\begin{tabular}{|clrrr|}
\hline \multirow{2}{*}{ Ítem } & & \multicolumn{3}{c|}{ Evaluador $\mathbf{2}$} \\
A & Evaluador 1 & Con Error Sin Error & p \\
& Con error & 1 & 1 & 1,0000 \\
B & Sin error & 2 & 28 & \\
& Con error & 5 & 1 & 0,6250 \\
& Sin error & 3 & 23 & \\
C & Con error & 0 & 0 & 1,0000 \\
& Sin error & 0 & 32 & \\
D & Con error & 0 & 0 & 1,0000 \\
& Sin error & 0 & 32 & \\
E & Con error & 2 & 0 & 1,0000 \\
& Sin error & 0 & 30 & \\
F & Con error & 4 & 0 & 1,0000 \\
& Sin error & 0 & 28 & \\
G & Con error & 0 & 0 & 1,0000 \\
& Sin error & 0 & 32 & \\
\hline
\end{tabular}

Prueba de McNemar.
D. No es posible determinar la vía de administración del medicamento, cuando existe ausencia de la vía de administración del o los medicamentos contenidos en ella, la información sea ilegible o esté incompleta.

E. No es posible determinar dosis diaria del medicamento, cuando existe ausencia de la dosis diaria del o los medicamentos contenidos en ella, la información sea ilegible o esté incompleta.

F. No es posible determinar duración del tratamiento, cuando existe ausencia del tiempo de duración del tratamiento del o los medicamentos contenidos en ella, la información sea ilegible o esté incompleta.

G. No es posible determinar cantidad total correcta del medicamento, cuando existe ausencia de la cantidad total correcta del o los medicamentos contenidos en ella, la información sea ilegible o esté incompleta. 
H. Prescripción repetida de un medicamento en la misma receta, cuando en una misma receta se repite un mismo medicamento (igual forma farmacéutica y concentración).

I. Uso de abreviaturas, símbolos o siglas en la prescripción de medicamentos.

J. Posee enmendaduras en las indicaciones de los medicamentos, cuando existe presencia de enmendaduras en la prescripción del fármaco, su concentración, dosis, duración de tratamiento y cantidad total recetada.

K. Ausencia de identificación del médico, cuando existe ausencia de los datos del médico (nombre y RUT), sean ilegibles o estén incompletos.

L. Ausencia de firma del médico.

\section{Tipos de errores en la preparación de recetas}

A. Medicamentos rotulados con indicaciones médicas incorrectas, cuando él o los medicamentos empacados contienen información errónea en relación a las indicaciones médicas prescritas en la receta médica.

B. Medicamentos rotulados con indicaciones médicas incompletas, cuando él o los medicamentos empacados contienen información incompleta en relación a las indicaciones médicas prescritas en la receta médica.

C. Medicamento empacado no corresponde al rotulado en la etiqueta, cuando él o los medicamentos empacados no coinciden con los rotulados en la parte externa del empaque.

D. Medicamento no corresponde a la forma farmacéutica recetada, cuando él o los medicamentos empacados no corresponden a las formas farmacéuticas indicadas en la receta médica.

E. No se preparó uno o más medicamentos prescritos en la receta médica, cuando faltó preparar uno o más de los medicamentos indicados en la receta médica.

F. Cantidad total del medicamento empacado incorrecto, cuando se empacan más o menos unidades de uno o más medicamentos en relación al total indicado en la receta médica.

G. Hay preparación de medicamentos que no están prescritos en la receta, cuando se preparan uno o más medicamentos que no venían indicados en la receta médica.

\section{Discusión}

Este trabajo adaptó pautas para registrar errores, tanto en las recetas médicas manuscritas como en el proceso de preparación de medicamentos en farmacia de atención de pacientes ambulatorios. La aplicación de pruebas estadísticas para evaluar la confiabilidad de cada pauta, da origen a dos instrumentos que brindan reproducibilidad en sus resultados, lo que hace que no sólo sean de utilidad en el Hospital de Nueva Imperial, sino que también en otros centros de similares características que requieran medir errores de medicación en las mencionadas etapas. Por el contrario, si los establecimientos difieren contextualmente, la metodología de adaptación y validación utilizada en este trabajo puede ser de mucha utilidad para dar origen a pautas de chequeo adaptadas a su propia realidad.

Desde la publicación del informe "To err is human: Building a safer health system" 10 en Estados Unidos de Norteamérica, la seguridad del paciente dentro de los hospitales ha sido un tema que ha tomado mayor relevancia, especialmente en lo que concierne a medicamentos. Esto ha impulsado una serie de medidas legislativas y reglamentarias por parte de las autoridades sanitarias, poniendo en marcha sistemas que permitirían informes de errores y prevención ${ }^{11}$. El desarrollo de técnicas y herramientas que permitan detectar este tipo de errores permiten un mejor uso de la información en beneficio de una adecuada gestión en el uso seguro de los medicamentos.

Dentro de las clasificaciones de errores de medicación, la elaborada por el grupo Ruiz-Jarabo 2000, ha sido ampliamente utilizada en diversos estudios, ya que constituye una herramienta útil para estandarizar la detección, análisis, clasificación y registro de los errores de medicación. Sin embargo, esta clasificación presenta ciertas limitaciones en la reproducibilidad de sus resultados, debido a la variabilidad en la interpretación de sus categorías, derivado, principalmente, de la subjetividad en el análisis que cada autor realiza ${ }^{8}$. En este punto, el registro de datos toma especial relevancia ya que a menudo los autores no publican las pautas u hojas de recolección utilizadas, muchas veces confeccionadas por los propios investigadores, en donde no se hace mención de algún proceso de validación de ellas.

Si bien este trabajo presenta como resultado 
Validación de instrumentos para medir errores de medicación - C. Alvarado et al

final dos pautas de chequeo para medir errores de medicación, también muestra un método para desarrollar instrumentos que brinden reproducibilidad en sus resultados. Entendiendo que cada Servicio de Farmacia de atención ambulatoria obedece a una realidad local diferente, y que puede variar de un país, ciudad o sistema de salud a otro, es recomendable volver a adaptar y validar las pautas si se van a utilizar en otros centros. De este modo, se podrán desarrollar estudios, en este tipo de errores de medicación, garantizando confiabilidad en sus resultados.

Agradecimientos: Se agradece la colaboración de los Químicos Farmacéuticos que contribuyeron con sus opiniones para la elaboración de las pautas de chequeo de este trabajo: Marly Ulloa (Hospital de Victoria), Jeaninna Saldivia (Hospital Dr. Juan Hepp Dubiau, Purranque), Georgina Ulloa (Hospital Dr. Augusto Riffart, Castro) y Fabiola Castillo (Hospital Base Valdivia). Al Centro de Excelencia CIGES (Capacitación, Investigación y Gestión para la Salud Basada en Evidencia) de la Universidad de La Frontera, por la entrega de las competencias necesarias para el desarrollo de este trabajo a través de su cuerpo docente en el marco de la Maestría en Epidemiología Clínica.

\section{Referencias}

1. Otero M. La gestión de riesgos en la prevención de los errores de medicación. En: Sociedad Española de Farmacia Hospitalaria, Curso de formación continuada en farmacoterapia de la SEFH. Madrid, España: Aran Ediciones; 2007. p. 111-51.

2. The National Coordinating Council for Medication
Error Reporting and Prevention, NCCMERP taxonomy of medication errors 2014. Disponible en: www.nccmerp.org/aboutMedErrors.html [Consultado el 16 de abril de 2014].

3. Lisby M, Nielsen L, Brock B, Mainz J. How are medication errors defined? A systematic literature review of definitions and characteristics. Int J Qual Health Care 2010; 22 (6): 507-18.

4. Ferner R. The epidemiology of medication errors: the methodological difficulties. Br J Clin Pharmacol; 2009; 67 (6): 614-20.

5. Aronson J. Medications errors: definitions and classification. Br J Clin Pharmacol 2009; 67 (6): 599-604.

6. Cheung K, Bouvy M, De Smet P. Medications errors: the importance of safe dispensing. Br J Clin Pharmacol 2009; 67 (6): 676-80.

7. Knudsen P, Herborg H, Mortensen A, Knudsen M, Hellebek A. Preventing medication errors in community pharmacy: frequency and seriousness of medication errors. Qual Saf Health Care 2007; 16: 291-6.

8. Otero M, Castaño B, Pérez M, Codina C, Tamés M, Sánchez T. Actualización de la clasificación de errores de medicación del grupo Ruiz Jarabo 2000. Farm Hosp 2008; 32 (1): 38-52.

9. Ministerio de Salud. Reglamento de farmacias, droguerías, almacenes farmacéuticos, botiquines y depósitos autorizados de 1984, DTO No 466. Marzo 12, 1985: 56-73.

10. Kohn L. Why do errors happen? En: Kohn LT, Corrigan JM, Donaldson MS, Editors, Committee on Quality of Health Care in America, To Err is Human: building a safer health system. Washington D.C, United States: National Academy Press 2000; p. 49-68.

11. Adubofour K, Keenan C, Daftary A, Mensah-Adubofour J, Dachman W. Strategies to reduce medication errors in ambulatory practice. J Natl Med Assoc 2004; 96 (12): 1558-64. 